\title{
Computed Tomography-Guided Biopsy for Potts Disease: An Institutional Experience from an Endemic Developing Country
}

\author{
Shashidhar Bangalore Kantharajanna ${ }^{1}$, Rajat Mahajan ${ }^{2}$ \\ ${ }^{1}$ Spine Services, Department of Orthopaedics, Vydehi Institute of Medical Sciences and Research Centre, Whitefield, Bangalore, India \\ ${ }^{2}$ Spine Services, Department of Orthopaedics, Indian Spinal Injuries Centre, New Delhi, India
}

We read article by Waqas et al. [1] entitled "Computed Tomography-Guided Biopsy for Potts Disease: An Institutional Experience from an Endemic Developing Country" with great interest. The authors have studied the diagnostic accuracy of computed tomography (CT) guided biopsy in Potts spine. The study is well written and the subject of the article is pertinent to our practice, since Potts spine is one the most common scenarios we have to deal with in our practice. The objective of the study is noteworthy and we have some questions for the authors:

(1) Previous studies have reported that the accuracy of the biopsy outcome depends on location of the lesion, with higher false negatives seen in cervical lesions by Rimondi et al. [2]. Did the authors find any variation in accuracy of the lesion according to anatomic location?

(2) It is mentioned that the biopsy was inconclusive in 22 patients $(24.2 \%)$, who received antitubercular therapy. What was the protocol for starting the anti-tubercular therapy in such inconclusive biopsies with doubtful diagnosis? Was the CT guided biopsy repeated?

(3) The type of needle influences the outcomes in diagnostic guided biopsy [3]. What was the type of needle used by the authors?

(4) There is a discrepancy in the data. The number acid fast bacilli culture positives is mentioned as $17.3 \%$ in the abstract and $13.2 \%$ in the discussion. We believe that this discrepancy arose as the authors have calculated the percentage by considering only the positives in the abstract section, while in the discussion they have considered all the 91 cases, including the inconclusive biopsies.

We appreciate the author's comments on these issues.

\section{Conflict of Interest}

No potential conflict of interest relevant to this article was reported.

\section{References}

1. Waqas M, Qadeer M, Faiz F, Alvi MA, Bari ME. Computed tomography-guided biopsy for Potts disease: an institutional experience from an endemic developing country. Asian Spine J 2015;9:394-8.

2. Rimondi E, Staals EL, Errani C, et al. Percutaneous CT-guided biopsy of the spine: results of 430 biopsies. Eur Spine J 2008;17:975-81.

3. Kim BJ, Lee JW, Kim SJ, Lee GY, Kang HS. Diagnostic yield of fluoroscopy-guided biopsy for infectious spondylitis. AJNR Am J Neuroradiol 2013;34:233-8.

\footnotetext{
Received Aug 2, 2015; Accepted Aug 8, 2015

Corresponding author: Shashidhar Bangalore Kantharajanna

Spine Services, Department of Orthopaedics, Vydehi Institute of Medical Sciences and Research Centre, \#82, EPIP Area, Nallurahalli, Near BMTC 18th Depot, Whitefield, Bengaluru, Karnataka 560066 Bengaluru, India Tel: +91-98-4552-9706, Fax:+91-172-2721133, E-mail: drshashidharbk@gmail.com
} 